\title{
Review
}

\section{Global impact of Torque teno virus infection in wild and domesticated animals}

\author{
Aldo Manzin ${ }^{1}$, Francesca Mallus ${ }^{1}$, Lisa Macera ${ }^{1,2}$, Fabrizio Maggi ${ }^{2}$, Sylvain Blois ${ }^{1}$ \\ ${ }^{1}$ Department of Biomedical Sciences, Clinical Microbiology and Virology Unit, University of Cagliari, Cagliari, Italy \\ 2 Virology Unit, Pisa University Hospital, Pisa, Italy
}

\begin{abstract}
Infection with Torque teno viruses (TTVs) is not restricted to humans. Different domestic and wild animal species are naturally infected with species-specific TTVs worldwide. Due to the global spread of the infection, it is likely that essentially all animals are naturally infected with species-specific TTVs, and that co-evolution of TTVs with their hosts probably occurred. Although TTVs are potentially related to many diseases, the evidence of the widespread infection in healthy human and nonhuman hosts raised doubts about their pathogenic potential. Nonetheless, their role as superimposed agents of other diseases or as triggers for impairment of immune surveillance is currently under debate. The possible contribution of animal TT viruses to interspecies transmission and their role as zoonotic agents are currently topics of discussion.
\end{abstract}

Key words: TT virus; anellovirus; animals; ssDNA; epidemiology.

J Infect Dev Ctries 2015; 9(6):562-570. doi:10.3855/jidc.6912

(Received 25 March 2015 - Accepted 05 May 2015)

Copyright $\odot 2015$ Manzin et al. This is an open-access article distributed under the Creative Commons Attribution License, which permits unrestricted use, distribution, and reproduction in any medium, provided the original work is properly cited.

\section{Introduction}

Anelloviruses (AVs) are a large group of small, non-enveloped viruses with a circular, single-stranded DNA genome of variable size, ranging from 2.1 to 3.8 $\mathrm{kb}$ [1-3]. Because of their high degree of genetic heterogeneity, AVs are divided into at least 11 genera, each of them including a various number of species [4]. AVs are ubiquitous and highly prevalent worldwide $[5,6]$. They infect their hosts with multiple and often divergent strains $[7,8]$ and are transmitted through multiple routes [9-15]. Infection with AVs probably occurs early in life [16] and leads to a progressive persistent infection of the natural host [17]. Human Torque teno virus (TTV) is the prototype of the Anelloviridae family, but a number of related viruses have been described in other mammalian hosts. Human TTV was originally reported in 1997 in a Japanese patient with post-transfusion, non-A-G hepatitis [18]. Since its discovery, a series of related human AVs have been characterized [19]. Furthermore, a series of closely related TTV-like viruses, and two distinct genera comprising viruses with smaller genome size and similar genetic organization, but only partial sequence homology, designated Torque teno midi virus (TTMDV) and Torque teno mini virus (TTMV), have been characterized [20-22]. A large number of AV species has also been reported in wild and domestic animals, including non-human primates, wild boars, badgers, pine martens, tupaias, rodents, bats, sea turtles, sea lions, livestock (pigs, sheep, cattle, camels, and poultry), and companion animals (cats and dogs) [3,5,23-29]. Indeed, since to date the presence of TTVs infecting mammalian species has been poorly investigated, it would not be surprising if all mammals were infected with species-specific TTVs. Human TTVs and TTVs infecting mammalian species have similar genomic and transcriptional organization, suggesting that co-evolution of TTVs with their hosts probably occurred [30]. However, AVs from different species share a low sequence homology, with genetic diversity varying between $35 \%$ (within genus) and $56 \%$ (among genera) [19]. In particular, AVs infecting animal species are highly divergent from those detected in humans, both in sequence composition and genome length, although phylogenetically some animal TTVs cluster with the human TTVs [31]. Despite over 17 years of investigation, little is known about the pathogenesis and possible disease associations with AV infections, in part due to the lack of a robust cell culture system for viral replication or a suitable small-animal model. 


\section{Discovery and characterization of TTV}

In 1997, Nishizawa et al., using representational difference analysis, isolated a sequence of 500 nucleotides from the blood of a patient suffering from a form of post-transfusion hepatitis of unknown etiology. The representative clone (N22) was found to originate from the genome of a non-enveloped, singlestranded DNA virus, provisionally named TTV, the initials of the index patient $[1,18]$. Subsequent cloning and sequencing experiments conducted by Okamoto et al. led to the characterization of a linear genome of $3,739 \mathrm{~kb}$, called TA278 [1]. In 1999, the presence of a GC-rich sequence of approximately $120 \mathrm{nt}$ was reported, allowing the recognition of the circular nature of the TTV genome with negative polarity [32]. The genomic full-length of the TA278 isolate was finally determined to be $3,853 \mathrm{nt}$, and the presence of the unique stem-and-loop structure in the GC-rich region was demonstrated $[1,2]$. Based on this and other structural and molecular characteristics that this virus shared with members of the family Circoviridae, and in particular with chicken anemia virus (CAV), TTV was initially recognized as the first human circovirus [32]. However, further studies showed no significant sequence identity between TTV and circovirus, and the virus was considered as the only representative of the new family Circinoviridae, from Latin circinatio, "circle" [2]. Shortly after the discovery, viral isolates were identified with genetic and structural characteristics similar to TTV, but with considerable genetic variability; among these, isolates PMV, SANBAN, and YONBAN [21,33] showed a nucleotide divergence greater than $50 \%$ compared to the prototype TA278. Subsequently, shorter sequences were isolated with genomic features intermediate between TTV and CAV; their characterization led to the identification of a new group of viruses named TTV-like mini virus (TTLMV, TTMV) [21]. Finally, in 2005, the International Committee on Taxonomy of Viruses (ICTV) proposed to classify TTMV and TTV into the new genus Anellovirus, and to assign to the acronym the meaning of Torque teno virus, from the Latin torque (necklace) and tenuis (narrow), referring to the characteristics of their genome [34]. TTV became the prototype of a large group of viral agents with similar genomic organization but a low sequence homology, isolated both in humans and in animals. In fact, in the following years, similar viruses were isolated in other species of vertebrates, including nonhuman primates (chimpanzees, macaques, tamarin monkeys, and douroucouli), pets (dogs and cats), livestock (pigs, cattle, sheep, camels, and poultry), and wild boars, badgers, pine martens, tupaias, rodents, bats, sea turtles, and sea lions [3,5,23-29,35]. The natural infection of pigs was first described by Leary et al. in 1999 [5]; the first complete genome of Torque teno sus virus (TTSuV) was described by Okamoto in 2002 [3]. In 17 years since discovery, over 200 complete or partial sequences of viral genomes like TTV were characterized in humans and more than 10 species of vertebrates. In 2009, the ICTV established the new family Anelloviridae, divided it into 11 genera, each one with a different number of species. Three other genera, Mutorquevirus, Nutorquevirus, and Xitorquevirus, which include newly discovered isolates, are currently under consideration by the ICTV for inclusion within the family (Table 1). Human TTVs belong to the genus Alphatorquevirus, which includes 29 species. In addition to TTV, in humans there are two other TTV-like species: TTMV and TTMDV, classified within the family Anelloviridae and belonging to the genera Betatorquevirus and Gammatorquevirus, respectively. TTVs infecting pigs (Sus domesticus) include four species described so far: Torque teno sus virus $1 \mathrm{a}$ and $1 \mathrm{~b}$ (TTSuV1a and TTSuV1b), belonging to the genus Iotatorquevirus,

Table 1. Current classification of the Anelloviridae family [4]

\begin{tabular}{ccc}
\hline Genus & Species & Host \\
\hline Alphatorquevirus & 29 & Humans, chimpanzees \\
Betatorquevirus & 12 & Humans, nonhuman primates \\
Deltatorquevirus & 1 & Tupaia \\
Epsilontorquevirus & 1 & Tamarin \\
Etatorquevirus & 2 & Cats \\
Gammatorquevirus & 15 & 2 \\
Iotatorquevirus & 2 & Humans, chimpanzees \\
Kappatorquevirus & 1 & Swine \\
Lambdatorquevirus & 1 & Swine \\
Thetatorquevirus & 1 & Sea lions \\
Zetatorquevirus & & Dogs \\
\hline
\end{tabular}


and Torque teno sus virus $\mathrm{k} 2 \mathrm{a}$ and $\mathrm{k} 2 \mathrm{~b}$ (TTSuVk2a and TTSuVk2b), the latter being the most recently discovered species, belonging to the genus Kappatorquevirus [36].

\section{Structure and genomic organization of TTV}

The virus appears as a spherical particle with icosahedral symmetry and a diameter of 30-50 nm. The buoyant density in cesium chloride was found to be $1.31-1.33 \mathrm{~g} / \mathrm{cm}^{3}$ for TTV in serum and $1.33-1.35$ $\mathrm{g} / \mathrm{cm}^{3}$ for TTV in feces [2]. Resistance to treatment with detergents, solvents, and dry heat confirmed the absence of a lipid envelope [37,38]. TTV proteins have not been well characterized yet; however, similar to $\mathrm{CAV}$, it is believed that the capsid is made up of a single structural protein. Sensitivity to DNase I, mung bean nuclease, and RNase $\mathrm{A}$, and resistance to restriction enzyme NdeI have proven the singlestranded nature of the viral DNA [2,37]. Hybridization with positive-sense RNA molecules and subsequent digestion has demonstrated the negative polarity of the viral DNA [2]. DNA size varies from 2.1 to $3.9 \mathrm{~kb}$, depending on the host species $[2,7,18,32]$; in general, the sizes of the animal AVs are smaller than those of human AVs. TTVs infecting humans and chimps have a genome size ranging from 3.7 to $3.9 \mathrm{~kb}$; the genome size of TTSuV (approximately $2.8 \mathrm{~kb}$ ) is intermediate between the size of human TTV and the size of the smallest AV identified so far $(<2.1 \mathrm{~kb})$, isolated in the cat [3]. Despite the variability in sequences and size, the various species of animal and human TTVs show similar genomic organization [3,24,39]. The genome consists of a coding region that contains at least three open reading frames (ORFs), and a non-translated region (UTR) of about $1.2 \mathrm{~kb}(0.8 \mathrm{~kb}$ in TTSuV), containing a GC-rich tract [40]. The highly conserved UTR region contains the TATA box and the polyA tail sequences and it is used as a transcription factor (Figure 1). The messenger RNAs (mRNAs) analyzed were transcribed from a plasmid containing the complete genome construct of TTV in COS1 cells [41]. Three spliced mRNAs of $3.0 \mathrm{~kb}, 1.2 \mathrm{~kb}$, and 1.0 $\mathrm{kb}$ with common 5'-and 3'-termini were recovered, and it was demonstrated that the splicing sites link distant ORFs to create two new ORFs capable of encoding 286 and 289 amino acids, respectively [41]. ORF1 encodes a product of about 700-770 amino acids, which is considered the viral capsid protein [3]. ORF2 codes for products of about 200 amino acids and in some genotypes it is divided into smaller ORF2a and ORF2b, the latter containing a CAV-like conserved amino acid motif, similar to protein- tyrosine phosphatase, that is probably involved in cellular and/or viral protein regulation and processing during natural infection [42]. The product from ORF3 is a protein of 285 amino acids with a serine-rich domain at the $\mathrm{C}$-terminus, and it resembles the structure and function of the non-structural protein $5 \mathrm{~A}$ of hepatitis $\mathrm{C}$ virus in suppressing the antiviral action of interferon [43]. A splicing-derived product of 105 amino acids, called TTV-derived apoptosis-inducing protein, induces apoptosis in hepatocellular carcinoma cells, but it is absent in swine AVs. It is likely that intra-genomic rearrangements and alternative splicing events are responsible for the generation of other ORFs, at least in some genotypes [44].

\section{Replication of TTV}

The distribution of TTV in the tissues of the natural hosts and their detection in a wide variety of biological samples suggest that AVs are able to replicate in different cell types, recognizing receptors distributed across different tissues [45-47]. For DNA replication, similar to circovirus and in analogy to the plant and bacterial viruses with circular singlestranded DNA, a rolling circle mechanism of replication has been proposed, leading to the formation of a circular, double-stranded intermediate [2]. As a DNA polymerase-coding sequence has never been demonstrated, it is believed that AVs, like most small DNA viruses, replicate their DNA using the DNA polymerase and the replication machinery of the host cell [48]. Replicating forms of the TTV genome have been detected in a variety of tissues, including lung, stimulated peripheral blood mononuclear cells, bone marrow, spleen, liver, pancreas, kidney, thyroid gland, and lymph nodes $[45,49,50]$. Furthermore, TTV was detected in the serum of most infected hosts. In bone marrow-derived cells, three types of mRNAs of TTV

Figure 1. Genomic organization of TTV. Nucleotide positions refer to the TA278 isolate [91].

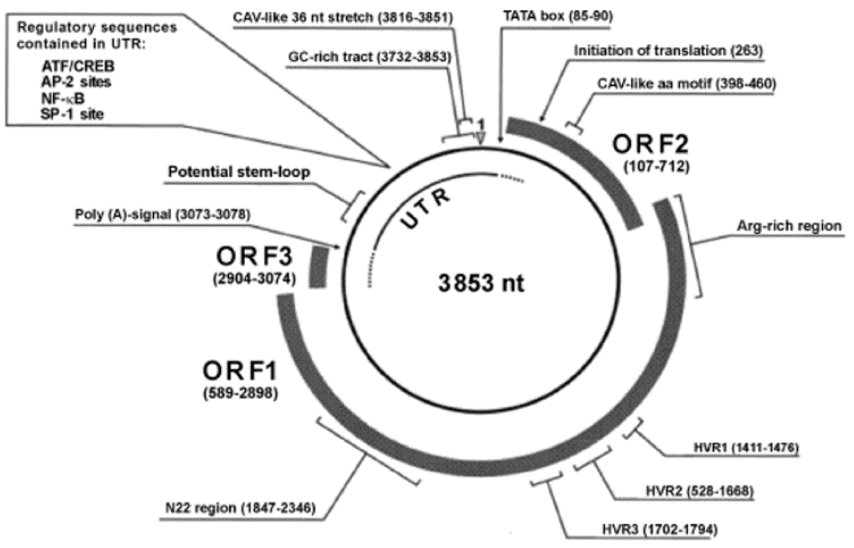


(2.9 kb, $1.2 \mathrm{~kb}$, and $1.0 \mathrm{~kb}$ ) have been detected and produced by alternative splicing, as well as by transfecting African green monkey cells with whole TTV genome; in this case, at least six proteins derived from three distinct mRNAs, expressed from two different start codons, have been produced.

\section{Genetic variability of TTV}

Using conserved UTR-specific primers for detection of TTV DNA, several variants with high genetic diversity have been detected $[7,21,32,35,51,52]$. Human TTVs belonging to the genus Alphatorquevirus are divided into at least 29 species and more than 40 genotypes and 70 subtypes, with nucleotide differences exceeding 50\% among species, more than $30 \%$ between genotypes and between $15 \%$ and $29 \%$ among subtypes. The sequence homology between human and swine TTV is below $45 \%$ [3,39,53]. Sequence homology between the TTSuV1 and TTSuV2 species was estimated to be around $56 \%$, nucleotide divergence being higher among isolates of TTSuV1 (30\%) compared to TTSuV2 isolates (15\%). Genetic variability is greater in ORF1, where at least three hypervariable regions characterized by several insertions and deletions and amino acid diversity among TTV isolates higher than $70 \%$ have been described. In TTV isolates belonging to the same genotype and in isolates belonging to different genotypes, at least 19 recombination sites have been identified, 13 of them located in the UTR [54]. Co-infection with strains of different genotypes is very frequent in humans $[8,55]$, as is co-infection with viral subtypes and multiple species of TTSuV in swine [56]. Finally, it is possible that the interspecies transmission of TTVs between humans and livestock or pet animals may contribute to the genetic variability of the virus [30].

\section{Animal TTVs}

TTV infection is not restricted to humans. Using highly conserved primers derived from the UTR of the TTV genome, a variety of TTV-like viruses have been detected in non-human primates and tupaias (tree shrews, Tupaia belangeri chinensis) [5,35,57]. The entire nucleotide sequences of species-specific TTVs that infect nonhuman primates, such as the chimpanzee (Pan troglodytes), Japanese macaque (Macaca fuscata), cotton-top tamarin (Saguinus oedipus), and douroucouli (Aotes trivirgatus) have been determined [24,35]. Human and simian TTVs share closely related genome organization and presumed transcriptional profiles, showing nearly $85 \%$ sequence similarity. However, it is now well established that TTV variants in nonhuman primates are species-specific $[5,23,24,35,58]$ and that TTVs from macaques and tamarins are increasingly divergent from TTV variants infecting humans and chimpanzees [35]. Recent evidence shows that simian TTV can infect humans, as it has been observed that approximately $10 \%$ of Japanese patients with liver diseases are infected with simian TTV, although the mode of transmission of the infection from animals to humans has not been demonstrated [59]. Furthermore, TTV DNA has been detected in serum samples obtained from wild and domesticated animals, including chickens, pigs, wild boars, camels, cats, dogs, pine martens, sea lions, cows, and sheep [3,5]. Even more recently, Nishiyama et al. reported the identification of diverse anelloviruses in several species of wild rodents; the viruses are highly prevalent in wood mice (Apodemus sylvaticus) and field voles (Microtus agrestis), detectable at a low frequency in bank voles (Myodes glareolus), but absent from house mice (Mus musculus) [28]. The presence of TTV in dogs (now named Torque teno canis virus) has been documented, and the full-length nucleotide sequence of Japanese and Chinese isolate strains has also been reported; results indicate that dogs are naturally infected with species-specific TTVs with small genomic sizes and suggest a global distribution of TTVs with extremely divergent genomic sequences and lengths in animals $[3,60]$. In Italian dogs, the presence of Torque teno canis virus has recently been checked for, and about $10 \%$ of the animals were found to be infected with highly divergent variants (unpublished data). Indeed, since TTV has been detected in different animals, it might be considered as a zoonotic infection. Hence, it is possible that the virus can be transmitted from humans to animals and vice versa. Many recent studies have focused on TTVs that infect pigs (TTSuV). The AVs that infect swine, domestic pigs, and wild boars are spread all over the world, regardless of the race, age, gender, and state of health of the animals [30,61-63]. A recent retrospective study has documented the circulation of the virus in Spanish farms since 1985 [64]. Globalization and international trade in live animals have been proven to play a crucial role in the spread of TTSuV [65]. In addition, the vast spread of the infection in swine and the absence of geographical clustering of the isolates have led some authors to speculate that the dissemination on a planetary scale may be partly due to the use of contaminated vaccines [13]. The global prevalence of infection varies 
between $16.8 \%$ and $100 \%$ for TTSuV1 [66-68], and between $31 \%$ and $90 \%$ for TTSuV2 $[63,66,69]$. Data related to infection with TTSuVk2b, the most recently identified species, are still limited. A study on 244 pig serum samples from 17 different countries reported an overall $40 \%$ prevalence of infection [36], whereas Blois et al. reported an $11.5 \%$ prevalence of TTSuVk2b in 721 Italian pigs [70]. The high prevalence of TTSuV in the swine population indicates the existence of effective mechanisms of transmission. Although the fecal-oral route is considered the primary mode of transmission, other ways may contribute to the dissemination of the virus. TTSuVs have been found in the feces and in the nasal secretions of piglets in their first week of life; the prevalence of infection increases with age, and the virus is shed more through nasal secretions than through fecal elimination [71,72]. TTSuVs are also found in seminal fluid, indicating the possibility of sexual transmission of infection [73]. TTSuV DNA has also been found in colostrum and stillborn piglets, indicating that vertical and transplacental or intrauterine transmission may contribute to the dissemination of the virus [14]. So, infection with TTSuV occurs early in life and leads to a progressive persistent infection, with viremia levels increasing with the age of the animals. Most tissues and pig organs, if not all (brain, lungs, kidneys, bone marrow, heart, spleen, and mesenteric and mediastinal lymph nodes), test positive at five weeks of age, reaching the highest prevalence at slaughter [46]. In particular, lymphoid tissues and $\mathrm{T}$ lymphocytes are hypothesized to be the target site and the target cells for TTSuV, respectively [74]. As in humans, TTV shows a high degree of genetic variability in the swine population, and the presence of co-infection with multiple species and viral subtypes is a frequent event $[39,56,75,76]$. The production of antibodies elicited against the viral capsid protein, which is associated with a reduction in viremia, has been demonstrated [77], but these antibodies seem unable to eradicate the infection, which in part could explain the persistence of the virus in the animal host. Finally, the intra-uterine infection of the fetus before the acquisition of mature immunocompetence could explain the establishment of a state of immunotolerance against TTSuV [46].

\section{Innocent bystanders or something else?}

$\mathrm{AVs}$ have been known to exist for almost two decades and, although much is known about their epidemiology, they are orphan viruses still in search of a disease. The high prevalence of infection in the general population and the capability to establish persistent infections raised doubts about their actual pathogenic potential, and some researchers have considered TTV a component of the human microbiota [78]. Although TTV has been potentially related to many diseases, there are only a few reports supporting the disease-inducing potential of TTV. A series of studies suggest a possible involvement of human TTVs in the pathogenesis of certain diseases, such as hepatitis [79], hematological disorders [80], respiratory diseases [12], rheumatic autoimmune diseases [81], and various malignant disorders. Regarding AVs infecting animals, most studies to date have focused on the role of TTSuV in pig diseases. Although TTSuVs were found at particularly high frequency in healthy animals [70,72], they are currently receiving more attention due to the latest results on disease association. In fact, TTSuVs are considered non-pathogenic by themselves, but there is increasing evidence that points to their influence on the development of some diseases or suggests that these viruses even affect disease outcome [66]. In particular, the co-infection with porcine circovirus type 2 (PCV2) and the associated porcine circovirus diseases deserve special attention [61]. First evidence highlighted the high prevalence of TTSuV2 infection in postweaning multisystemic syndrome (PMWS), although in most cases, such co-infection does not lead to a disease; however, it is subclinical, suggesting that the disease association is possibly a matter of viral load [66]. Recent reports showed that porcine TTV partially contributed to inducing porcine reproductive and respiratory syndrome, porcine dermatitis and nephropathy syndrome, and hepatitis [82,83]. The latest research indicating that TTSuV2 but not TTSuV1 seems to benefit from the host's disease status suggests that TTSuVs may act as triggers for diseases that cause immune system impairment. In this context, the level of TTSuV2 viremia may be associated with the level of immunocompetence of the animals, resulting in uncontrolled in PMWS animals, which are known to be immunocompromised [66]. Similarly, TTV DNA load in infected humans appears to correlate with the level of immune competence of the host $[84,85]$. Some authors have recently suggested that the expansion of members of AVs in the human microbiome and the increasing viral load in plasma during immunosuppressive therapy could be used to predict and monitor immune competence [86]. The association between the single infection with TTSuV or the co-infection with PCV2 and abortion in pigs has not been confirmed [16]. However, a recent 
study carried out using pigs infected with the hepatitis $\mathrm{E}$ virus has shown a correlation between TTSuV and the increased risk of developing severe hepatitis in animals co-infected with PCV2 [87]. More recently, a high prevalence of TTSuV1, but not TTSuV2, in pigs suffering from porcine respiratory disease complex has been shown [88]. As for other porcine pathogens $(\mathrm{PCV})$, it is therefore possible to speculate that the TTSuV species are distinct not only from the genetic point of view, but also for their different pathogenic potential.

\section{Conclusions}

Since their identification about 20 years ago, many studies have been conducted on human and animal TTVs, in order to assess the extent of dissemination of the infection and the pathogenic potential of the viruses $[30,89]$. It is likely that such viruses would be considered as components of the host microbiota and, as such, unable to cause disease directly, but rather available to be engaged in physiological processes and to modulate the response of the organism to other pathogens. It was recently suggested that the dynamics of replication of human TTVs can correlate with the immune competence of the host, and that the viral load can be considered as a possible biomarker of immune responsiveness [84]. It has been also proposed that animal AVs, especially TTSuVs, could be utilized as a model to evaluate the dynamics and the effects of global trade on viral heterogeneity, and to understand how live animal movement affects virus evolution [65]. Furthermore, it was hypothesized that TTV could be a more appropriate indicator of viral pathogens in drinking water than currently used indicator systems based solely on bacteria, thus providing an efficient indicator system for viral pathogen risk [90]. This indicator would have broad application to drinking water utilities and would provide a better means to assess viral risk and protect public health, especially in developing countries. Furthermore, TTSuV has been detected in commercial pig products including vaccines, enzymes for laboratory use, and human drugs containing components of porcine origin [13]. Such evidence would indicate the need to screen these products for the presence of these viruses. Finally, it has been reported that the prevalence of TTV is geographically variable. Could such a large variability influence the behavior of the infection at an individual level? Could the chronic exposure to this virus in a particular geographical area, especially in developing countries, be a source of repetitive pathogenicity for hosts? These and other epidemiological and biological aspects of animal AVs should be studied with large comparative and prospective studies.

\section{Acknowledgements}

This review was written in honor of Professor Pietro Cappuccinelli: a friend, a colleague, a great scientist. This work was funded by grant from Regione Sardegna (Italy) Nr. F71J11000710002.

\section{References}

1. Okamoto H, Nishizawa T, Kato N, Ukita M, Ikeda H, Iizuka Y, Miyakawa Y, Mayumi M (1998) Molecular cloning and characterization of a novel DNA virus (TTV) associated with posttransfusion hepatitis of unknown etiology. Hepatol Res 10: 1-16.

2. Mushahwar IK, Erker JC, Muerhoff AS, Leary TP, Simons JN, Birkenmeyer LG, Chalmers ML, Pilot-Matias TJ, Dexai SM (1999) Molecular and biophysical characterization of TT virus: evidence for a new virus family infecting humans. Proc Natl Acad Sci U S A 96: 3177-3182.

3. Okamoto H, Takahashi M, Nishizawa T, Tawara A, Fukai K, Muramatsu U, Naito Y, Yoshikawa A (2002) Genomic characterization of TT viruses (TTVs) in pigs, cats and dogs and their relatedness with species-specific TTVs in primates and tupaias. J Gen Virol 83: 1291-1297.

4. International Committee on Taxonomy of Viruses. Available: http://www.ictvonline.org/virusTaxonomy.asp. Accessed 15 March, 2015.

5. Leary TP, Erker JC, Chalmers ML, Desai SM, Mushahwar IK (1999) Improved detection systems for TT virus reveal high prevalence in humans, non-human primates and farm animals. J Gen Virol 80: 2115-2120.

6. Maggi F, Fornai C, Morrica A, Casula F, Vatteroni ML, Marchi S, Ciccorossi P, Riente L, Pistello M, Bendinelli M (1999) High prevalence of TT virus viremia in Italian patients, regardless of age, clinical diagnosis, and previous interferon treatment. J Infect Dis 180: 838-842.

7. Okamoto H, Takahashi M, Nishizawa T, Ukita M, Fukuda M, Tsuda F, Miyakawa Y, Mayumi M (1999) Marked genomic heterogeneity and frequent mixed infection of TT virus demonstrated by PCR with primers from coding and noncoding regions. Virology 259: 428-436.

8. de Arruda Leme RA, Ribeiro J, Alfieri AF, Alfieri AA (2013) Simultaneous infection with distinct strains of torque teno sus virus (TTSuV) in healthy slaughter-age pigs. Vet Res Commun 37: 183-186.

9. Inami T, Konomi N, Arakawa Y, Abe K (2000) High prevalence of TT virus DNA in human saliva and semen. J Clin Microbiol 38: 2407-2408.

10. Itoh Y, Takahashi M, Fukuda M, Shibayama T, Ishikawa T, Tsuda F, Tanaka T, Nishizawa T, Okamoto H (2000) Visualization of TT virus particles recovered from the sera and feces of infected humans. Biochem Biophys Res Commun 279: 718-724.

11. Deng X, Terunuma H, Handema R, Sakamoto M, Kitamura T, Ito M, Akahane Y (2000) Higher prevalence and viral load of TT virus in saliva than in the corresponding serum: another possible transmission route and replication site of TT virus. J Med Virol 62: 531-537. 
12. Maggi F, Pifferi M, Fornai C, Andreoli E, Tempestini E, Vatteroni M, Presciuttini S, Marchi S, Pietrobelli A, Boner A, Pistello M, Bendinelli M (2003) TT virus in the nasal secretions of children with acute respiratory diseases: relations to viremia and disease severity. J Virol 77: 24182425.

13. Kekarainen T, Martínez-Guinó L, Segalés J (2009) Swine torque teno virus detection in pig commercial vaccines, enzymes for laboratory use and human drugs containing components of porcine origin. J Gen Virol 90: 648-653.

14. Martínez-Guinó L, Kekarainen T, Segalés J (2009) Evidence of Torque teno virus (TTV) vertical transmission in swine. Theriogenology 71: 1390-1395.

15. Zehender G, Manzin A, De Maddalena C, Colasante C, Solforosi L, Corsi F, Bianchi-Bosisio A, Girotto M, Schirru I, Russo U, Galli M, Clementi M (2001) Molecular epidemiology of TT virus in Italy and phylogenesis of viral isolates from subjects at different risk for parenteral exposure. J Med Virol 63: 76-84.

16. Martínez-Guinó L, Kekarainen T, Maldonado J, Aramouni M, Llorens A, Segales J (2010) Torque teno sus virus (TTV) detection in aborted and slaughterhouse collected fetuses. Theriogenology 74: 277-281.

17. Brassard J, Gagné MJ, Leblanc D (2013) Real-Time PCR study of the infection dynamics of Torque teno sus viruses in naturally infected pigs from nursery to slaughterhouse. Vet J 197: 506-508.

18. Nishizawa $T$, Okamoto $H$, Konishi $K$, Yoshizawa $H$, Miyakawa Y, Mayumi M (1997) A novel DNA virus (TTV) associated with elevated transaminase levels in posttransfusion hepatitis of unknown etiology. Biochem Biophys Res Commun 241: 92-97.

19. Biagini P, Bendinelli M, Hino S, Kakkola L, Mankertz A, Niel C, Okamoto H, Raidal S, Teo CG, Todd D (2011) Anelloviridae. In King AMQ, Adams MJ, Carstens EB, Lefkowitz EJ, editors. Virus Taxonomy, 9th Report of the ICTV. London: Elsevier Academic Press. 331-341.

20. Biagini P, Gallian P, Attoui H, Touinssi M, Cantaloube JF, de Micco P, de Lamballerie X (2001) Genetic analysis of fulllength genomes and subgenomic sequences of TT virus-like mini virus human isolates. J Gen Virol 82: 379-383.

21. Takahashi K, Hijikata M, Samokhvalov EI, Mishiro S (2000) Full or near full length nucleotide sequences of TT virus variants (types SANBAN and YONBAN) and the TT viruslike mini virus. Intervirology 43: 119-123.

22. Umemura T, Yeo AE, Sottini A, Moratto D, Tanaka Y, Wang RY, Shih JW, Donahue P, Primi D, Alter HJ (2001) SEN virus infection and its relationship to transfusion-associated hepatitis. Hepatology 33: 1303-1311.

23. Abe K, Inami T, Ishikawa K, Nakamura S, Goto S (2000) TT virus infection in nonhuman primates and characterization of the viral genome: identification of simian TT virus isolates. J Virol 74: 1549-1553.

24. Okamoto H, Nishizawa T, Takahashi M, Tawara A, Peng Y, Kishimoto J, Wang Y (2001) Genomic and evolutionary characterization of TT virus (TTV) in tupaias and comparison with species-specific TTVs in humans and non-human primates. J Gen Virol 82: 2041-2050.

25. Thom K, Morrison C, Lewis JC, Simmonds P (2003) Distribution of TT virus (TTV), TTV-like minivirus, and related viruses in humans and nonhuman primates. Virology 306: 324-333.
26. Al-Moslih MI, Perkins H, Hu YW (2007) Genetic relationship of Torque Teno virus (TTV) between humans and camels in United Arab Emirates (UAE). J Med Virol 79: 188-191.

27. Ng TF, Suedmeyer WK, Wheeler E, Gulland F, Breitbart M (2009) Novel anellovirus discovered from a mortality event of captive California sea lions. J Gen Virol 90: 1256-1261.

28. Nishiyama S, Dutia BM, Stewart JP, Meredith AL, Shaw DJ, Simmonds P, Sharp CP (2014) Identification of novel anelloviruses with broad diversity in UK rodents. J Gen Virol 95: 1544-1553.

29. Cibulski SP, Teixeira TF, de Sales Lima FE, do Santos HF, Franco AC, Roehe PM (2014) A novel Anelloviridae species detected in Tadarida brasiliensis bats: First sequence of a Chiropteran Anellovirus. Genome Announc 2: e01028-14.

30. Okamoto H (2009) TT viruses in animals. Curr Top in Microbiol Immunol 331: 35-52.

31. Biagini P (2009) Classification of TTV and Related Viruses (Anelloviruses). In de Villiers EM, zur Hausen H, editors. TT Viruses. The Still Elusive Human Pathogens. Berlin: Springer-Verlag. 21-33.

32. Miyata H, Tsunoda H, Kazi A, Yamada A, Khan MA, Murakami J, Kamahora T, Shiraki K, Hino S (1999) Identification of a novel GC-Rich 113-nucleotide region to complete the circular, single stranded DNA genome of TT virus, the first human circovirus. J Virol 73: 3582-3586.

33. Hallet RL, Clewley JP, Bobet F, McKiernan PJ, Teo CG (2000) Characterization of a highly divergent TT virus genome. J Gen Virol 81: 2273-2279.

34. Todd D, Bendinelli M, Biagini P, Hino S, Mankertz A, Mishiro S, Niel C, Okamoto H, Raidal S, Ritchie BW, Teo CC (2005) Virus Taxonomy VIIIth Report of the International Committee on Taxonomy of Viruses. London: Academic Press. 335-341.

35. Okamoto H, Nishizawa T, Tawara A, Peng Y, Takahashi M, Kishimoto J, Tanaka T, Miyakawa Y, Mayumi M (2000) Species-specific TT viruses in humans and nonhuman primates and their phylogenetic relatedness. Virology 277: 368-378.

36. Cornelissen-Keijsers V, Jiménez-Melsió A, Sonnemans D, Cortey M, Segalés J, van den Bor E, Kekarainen T (2012) Discovery of a novel Torque teno sus virus species: genetic characterization, epidemiological assessment, and disease association. J Gen Virol 93: 2682-2691.

37. Okamoto H, Akahane Y, Ukita M, Fukuda M, Tsuda F, Miyakawa Y, Mayumi M (1998) Fecal excretion of a nonenveloped DNA virus (TTV) associated with posttransfusion non-A-G hepatitis. J Med Virol 56: 128-132.

38. Ukita M, Okamoto H, Kato N, Miyakawa Y, Mayumi M (1999) Excretion into bile of a novel unenveloped DNA virus (TT virus) associated with acute and chronic non-A-G hepatitis. J Infect Dis 179: 1245-1248.

39. Niel C, Diniz-Mendes L, Devalle S (2005) Rolling-circle amplification of Torque teno virus (TTV) complete genomes from human and swine sera and identification of a novel swine TTV genogroup. J Gen Virol 86: 1343-1347.

40. Kamada K, Kamahora T, Kabat P, Hino S (2004) Transcriptional regulation of TT virus: promoter and enhancer regions in the 1.2-kb noncoding region. Virology 10: 341348.

41. Kamahora T, Hino S, Miyata H (2000) Three spliced mRNAs of TT virus transcribed from a plasmid containing the entire genome in COS1 cells. J Virol 74: 9980-9986. 
42. Zheng H, Ye L, Fang X, Li B, Wang Y, Xiang X, Kong L, Wang W, Zeng Y, Ye L, Wu Z, She Y, Zhou X (2007) Torque teno virus (SANBAN isolate) ORF2 protein suppresses NF-kappaB pathways via interaction with IkappaB kinases. J Virol 81: 11917-11924.

43. Asabe S, Nishizawa T, Iwanari H, Okamoto H (2001) Phosphorylation of serine-rich protein encoded by open reading frame 3 of the TT virus genome. Biochem Biophys Res Commun 286: 298-304.

44. Leppik L, Gunst K, Lehtinen M, Dillner J, Streker K, de Villiers EM (2007) In vivo and in vitro intragenomic rearrangement of TT viruses. J Virol 81: 9346-9356.

45. Okamoto H, Nishizawa T, Takahashi M, Asabe S, Tsuda F, Yoshikawa A (2001) Heterogeneous distribution of TT virus of distinct genotypes in multiple tissues from infected humans. Virology 288: 358-368.

46. Aramouni M, Segalés J, Cortey M, Kekarainen T (2010) Agerelated tissue distribution of swine Torque teno sus virus 1 and 2. Vet Microbiol 146: 350-353.

47. Zhu CX, Yuan CL, Cui L, Yu Y, Liu RA, Zhao W, Hua XG (2012) Molecular detection of Torque teno sus virus from tissues samples of sick pigs in China. Virus Res 165: 225230.

48. Kakkola L, Tommiska J, Boele LC, Miettinen S, Blom T, Kekarainen T, Qiu J, Pintel D, Hoeben RC, Hedman K, Söderlund-Venermo M (2007) Construction and biological activity of a full-length molecular clone of human Torque teno virus (TTV) genotype 6. FEBS J 274: 4719-4730.

49. Bando M, Ohno S, Oshikawa K, Takahashi M, Okamoto H, Sugiyama Y (2001) Infection of TT virus in patients with idiopathic pulmonary fibrosis. Respir Med 95: 935-942.

50. Mariscal LF, López-Alcorocho JM, Rodríguez-Iñigo E, OrtizMovilla N, de Lucas S, Bartolomé J, Carreño V (2002) TT virus replicates in stimulated but not in nonstimulated peripheral blood mononuclear cells. Virology 301: 121-129.

51. Khudyakov YE, Cong ME, Nichols B, Reed D, Dou XG, Viazov SO, Chang J, Fried MW, Williams I, Bower W, Lambert S, Purdy M, Roggendorf M, Fields HA (2000) Sequence heterogeneity of TT virus and closely related viruses. J Virol 74: 2990-3000.

52. Okamoto H, Kato N, lizuka H, Tsuda F, Miyakawa Y, Mayumi M (1999) Distinct genotypes of a nonenveloped DNA virus associated with posttransfusion non-A to $G$ hepatitis (TT virus) in plasma and peripheral blood mononuclear cells. J Med Virol 57: 252-258.

53. Biagini P, Uch R, Belhouchet M, Attoui H, Cantaloube JF, Brisbarre N, de Micco P (2007) Circular genomes related to anelloviruses identified in human and animal samples by using a combined rolling-circle amplification/sequenceindependent single primer amplification approach. J Gen Virol 88: 2696-2701.

54. Worobey M (2000) Extensive homologous recombination among widely divergent TT viruses. J Virol 74: 7666-7670.

55. Maggi F, Andreoli E, Lanini L, Fornai C, Vatteroni ML, Pistello M, Presciuttini S, Bendinelli M (2005) Relationships between total plasma load of torque teno virus (TTV) and TTV genogroups carried. J Clin Microbiol 43: 4807-4810.

56. Liu J, Guo L, Zhang L, Wei Y, Huang L, Wu H, Liu C (2013) Three new emerging subgroups of torque teno sus viruses (TTSuVs) and co-infection of TTSuVs with porcine circovirus type 2 in China. Virol J 10: 189.
57. Verschoor EJ, Langenhuijzen S, Heeney JL (1999) TT viruses (TTV) of non-human primates and their relationship to the human TTV genotypes. J Gen Virol 80: 2491-2499.

58. Okamoto H, Fukuda M, Tawara A, Nishizawa T, Itoh Y, Hayasaka I, Tsuda F, Tanaka T, Miyakawa Y, Mayumi M (2000) Species-specific TT viruses and cross-species infection in nonhuman primates. J Virol 74: 1132-1139.

59. Iwaki Y, Aiba N, Tran HT, Ding X, Hayashi S, Arakawa Y, Sata T, Abe K (2003) Simian TT virus (s-TTV) infection in patients with liver diseases. Hepatol Res 25: 135-142.

60. Lan D, Hua X, Cui L, Luo X, Liu Z, San T, Zhu C, Zhao W, Yang Z (2011) Sequence analysis of a Torque teno canis virus isolated in China. Virus Res 160: 98-101.

61. Kekarainen T, Segalés J (2012) Torque teno sus virus in pigs: an emerging pathogen? Transbound Emerg Dis 59: 103-108.

62. Cadar D, Kiss T, Adám D, Csagola A, Novosel D, Tuboly T (2013) Phylogeny, spatio-temporal phylodynamics and evolutionary scenario of Torque teno sus virus 1 (TTSuV1) and 2 (TTSuV2) in wild boars: fast dispersal and high genetic diversity. Vet Microbiol 166: 200-213.

63. Mei M, Zhu L, Xu Z, Zhao L, Zhou Y, Wu Y, Li S, Wei H, Guo W (2013) Molecular investigation of Torque teno sus virus in geographically distinct porcine breeding herds of Sichuan, China. Virol J 10: 161.

64. Segalés J, Martínez-Guinó L, Cortey M, Navarro N, Huerta E, Sibila M, Pujols J, Kekarainen T (2009) Retrospective study on swine Torque teno virus genogroups 1 and 2 infection from 1985 to 2005 in Spain. Vet Microbiol 134: 199-207.

65. Cortey M, Pileri E, Segalés J, Kekarainen T (2012) Globalisation and global trade influence molecular viral population genetics of Torque teno sus viruses 1 and 2 in pigs. Vet Microbiol 156: 81-87.

66. Kekarainen T, Sibila M, Segalés J (2006) Prevalence of swine Torque teno virus in post-weaning multisystemic wasting syndrome (PMWS)-affected and non- PMWS-affected pigs in Spain. J Gen Virol 87: 833-837.

67. Brink M, Stahl K, Masembe C, Okurut AR, Berg M, Blomström AL (2012) First time molecular detection and phylogenetic relationships of torque teno sus virus 1 and 2 in domestic pigs in Uganda: further evidence for a global distribution. Virol J 9: 39.

68. Xiao CT, Giménez-Lirola L, Huang YW, Meng XJ, Halbur PG, Opriessnig T (2012) The prevalence of Torque teno sus virus (TTSuV) is common and increases with the age of growing pigs in the United States. J Virol Methods 183: 4044.

69. Lee SS, Sunyoung S, Jung H, Shin J, Lyoo YS (2010) Quantitative detection of porcine Torque teno virus in Porcine circovirus-2-negative and Porcine circovirusassociated disease-affected pigs. J Vet Diagn Invest 22: 261264.

70. Blois S, Mallus F, Liciardi M, Pilo C, Camboni T, Macera L, Maggi F, Manzin A (2014) High prevalence of co-infection with multiple Torque teno sus virus species in Italian pig herds. PLoS ONE 9: e113720.

71. Brassard J, Gagné MJ, Lamoureux L, Inglis GD, Leblanc D, Houde A (2008) Molecular detection of bovine and porcine Torque teno virus in plasma and feces. Vet Microbiol 126: 271-276.

72. Sibila M, Martínez-Guinó L, Huerta E, Llorens A, Mora M, Grau-Roma L, Kekarainen T, Segales J (2009) Swine torque teno virus (TTV) infection and excretion dynamics in conventional pig farms. Vet Microbiol 139: 213-218. 
73. Kekarainen T, Lopez-Soria S, Segalés J (2007) Detection of swine Torque teno virus genogroups 1 and 2 in boar sera and semen. Theriogenology 68: 966-971.

74. Lee Y, Lin CM, Jeng CR, Pang VF (2014) Detection of torque teno sus virus 1 and 2 in porcine tissues by in situ hybridization using multi-strained pooled probes. Vet Microbiol 172: 390-399.

75. Leme RA, Ribeiro J, Alfieri AF, Alfieri AA (2013) Simultaneous infection with distinct strains of torque teno sus virus (TTSuV) in healthy slaughter-age pigs. Vet Res Commun 37: 183-186.

76. Cortey M, Macera L, Segalés J, Kekarainen T (2011) Genetic variability and phylogeny of Torque teno sus virus 1 (TTSuV1) and 2 (TTSuV2) based on complete genomes. Vet Microbiol 148: 125-131.

77. Huang YW, Harrall KK, Dryman BA, Beach NM, Kenney SP, Opriessnig T, Vaughn EM, Roof MB, Meng XJ (2011) Expression of the putative ORF1 capsid protein of Torque teno sus virus 2 (TTSuV2) and development of Western blot and ELISA serodiagnostic assays: correlation between TTSuV2 viral load and IgG antibody level in pigs. Virus Res 158: 79-88.

78. Simmonds P, Prescott LE, Logue C, Davidson F, Thomas AE, Ludlam CA (1999) TT virus-- part of the normal human flora? J Infect Dis 180: 1748-1750.

79. Asim M, Singla R, Gupta RK, Kar P (2010) Clinical \& molecular characterization of human TT virus in different liver diseases. Indian J Med Res 131: 545-554.

80. Focosi D, Maggi F, Albani M, Macera L, Ricci V, Gragnani S, Di Beo S, Ghimenti M, Antonelli G, Bendinelli M, Pistello M, Ceccherini-Nelli L, Petrini M (2010) Torque teno virus viremia kinetics after autologous stem cell transplantation are predictable and may serve as a surrogate marker of functional immune reconstitution. J Clin Virol 47: 189-192.

81. Gergely P Jr, Perl A, Poor G (2006) Possible pathogenic nature of the recently discovered TT virus: does it play a role in autoimmune rheumatic diseases? Autoimmun Rev 6: 5-9.

82. Krakowka S, Hartunian C, Hamberg A, Shoup D, Rings M, Zhang Y, Allan G, Ellis JA (2008) Evaluation of induction of porcine dermatitis and nephropathy syndrome in gnotobiotic pigs with negative results for porcine circovirus type 2. Am J Vet Res 69:1615-1622.

83. Aramouni M, Segalés J, Sibila M, Martin-Valls GE, Nieto D, Kekarainen T (2011) Torque teno sus virus 1 and 2 viral loads in postweaning multisystemic wasting syndrome (PMWS) and porcine dermatitis and nephropathy syndrome (PDNS) affected pigs. Vet Microbiol 153: 377-381.
84. Touinssi M, Gallian P, Biagini P, Attoui H, Vialettes B, Berland Y, Tamalet C, Dhiver C, Ravaux I, De Micco P, De Lamallerie X (2001) TTV virus infection: prevalence of elevated viraemia and arguments for the immune control of viral load. J Clin Virol 21: 135-141.

85. Maggi F, Pifferi M, Michelucci A, Albani M, Sbranti S, Lanini L, Simi P, Macchia P, Pistello M, Bendinelli M (2011) Torque teno virus viremia load size in patients with selected congenital defects of innate immunity. Clin Vaccine Immunol 18: 692-694.

86. De Vlaminck I, Khush KK, Strehl C, Kohli B, Luikart H, Neff NF, Okamoto J, Snyder TM, Cornfield DN, Nicolls MR, Weill D, Bernstein D, Valantine HA, Quake SR (2013) Temporal response of the human virome to immunosuppression and antiviral therapy. Cell 155: 11781187.

87. Savic B, Milicevic V, Bojkovski J, Kureljusic B, Ivetic V, Pavlovic I (2010) Detection rates of the swine torque teno viruses (TTVs), porcine circovirus type 2 (PCV2) and hepatitis $\mathrm{E}$ virus (HEV) in the livers of pigs with hepatitis. Vet Res Commun 34: 641-648.

88. Rammohan L, Xue L, Wang C, Chittick W, Ganesan S, Ramamoorthy S (2012) Increased prevalence of torque teno viruses in porcine respiratory disease complex affected pigs. Vet Microbiol 157: 61-68.

89. Okamoto H (2009) History of discoveries and pathogenicity of TT viruses. Curr Top Microbiol Immunol 331: 1-20.

90. Griffin JS, Plummer JD, Long SC (2008) Torque teno virus: an improved indicator for viral pathogens in drinking waters. Virol J 5: 112.

91. Bendinelli M, Pistello M, Maggi F, Fornai C, Freer G, Vatteroni ML (2001) Molecular properties, biology, and clinical implications of TTV virus, a recently identified widespread infectious agent of humans. Clin Microbiol Rev 14: 98-113.

\section{Corresponding author}

Aldo Manzin

Department of Biomedical Sciences, Clinical Microbiology and Virology Unit

Azienda Ospedaliero-Universitaria Cagliari - Policlinico Duilio

Casula, S.S. 554, Bivio per Sestu

I-09042 Monserrato (CA), Italy

Phone/Fax: +39070 51096350

Email: aldomanzin@medicina.unica.it

Conflict of interests: No conflict of interests is declared. 